\title{
Beschleunigungsverletzung der Halswirbelsäule
}

$D_{i}$ Beschleunigungsverletzung der Halswirbelsäule stellt ein besonderes Problem in Diagnostik und Therapie dar. Die Relevanz der Thematik ergibt sich aus der großen Zahl der Betroffenen, die über Beschwerden im Kopf-Hals-Schulter-Bereich nach Beschleunigungsverletzung klagen und einen chronischen Verlaufzeigen. Oft sind die Beschwerden nicht eindeutig faßbar und ein Funktionsdefizit nur schwer zu erkennen und zu objektivieren. Biomechanische Studien versuchen, den Verletzungsmechanismus nachzuahmen. Gleichwohl verzeichnen wir bei dem heutigen Stand der Forschung unterschiedliche Aussagen zum Ablauf des Verletzungsmechanismus sowie der daraus folgenden funktionellen oder gar strukturellen Störungen. Entsprechend basieren unsere Vorstellungen traumainduzierter Funktionsstörungen auf nicht eindeutig zu belegende Hypothesen.

Zur Beurteilung des jeweiligen Individualfalls muß eine konsequente diagnostische Analyse gefordert werden, die wegen unterschiedlicher Lokalisation und Ausprägung einer möglichen Funktionsstörung schwierig ist. Zudem wird die Beschwerdesymptomatik stets von subjektiven Parametern geprägt. Insbesondere lassen sich Befindlichkeitsstörungen, die bei einem somatischen Ansatz durch vegetative Begleitreaktionen erklärt werden mögen, nicht sicher von psychischen Faktoren oder auch einem hinter der geschilderten Beschwerdesymptomatik stehenden Entschädigungsbegehren unterscheiden. Selbst bei somatischen Störungen stehen wir vor dem Problem, daß übliche, tradierte Untersuchungstests in ihrer Validität nicht belegt sind und die Trennschärfe der verschiedenen diagnostischen Analyseverfahren mangelhaft ist.

Eine frühzeitige, klare Einordnung der Beschwerdesymptomatik beugt der Chronifizierung vor. Zudem kennen wir heute prognostische Faktoren, die eine Chronifizierungstendenz begründen.

Mit diesem Themenheft soll versucht werden, neuroanatomische Gesichtspunkte zu beleuchten, die Erklärungen für die oft unklare Beschwerdesymptomatik liefern. Zugleich soll versucht werden, mit Hilfe einer konsequenten, diagnostischen Analysestaffel, eine Richtschnur für das praktische Vorgehen zu geben.

Eine abgestufte, angemessene Therapie ist die Grundlage für eine zügige Rehabilitation, die nach funktionellen Grundsätzen ausgerichtet ist. Bei Versagen einzelner Maßnahmen ist rechtzeitig eine interdisziplinäre Abklärung einzuleiten. Aufjeden Fall muß eine Polypragmasie vermieden werden, die $z u$ einer Beschwerdepersistenz beiträgt.

Die gutachterliche Situation ist zum einen von den Problemen der diagnostischen Analyse geprägt, zum anderen von der oft unklaren, subjektiven Beschwerdesymptomatik mit nur schwer zu verifizierender und auf das angeschuldigte Trauma zurückzuführender Funktionsstörung. Die Grundsätze der Beurteilung können heute nicht mehr an pauschalen Vorgaben festgemacht werden, sondern bedürfen in besonderem Maße der Abwägung zwischen Unfallhergang und geklagter Beschwerdesymptomatik. Dabei ist es nicht vertretbar, lediglich auf die Höhe der Geschwindigkeitsdifferenzen oder symptomarme Intervalle abzustellen, wie dies mitunter simplifizierend gemacht wird.

So will dieses Heft einige neuere Aspekte in Diagnose, Therapie und Begutachtung beisteuern, um mit verbesserter Differenzierung dem nach wie vor wissenschaftlich nicht befriedigend geklärten Problem der Funktionsstörungen bei Beschleunigungsverletzung besser gerecht zu werden.

\section{J. Grifka}

